\title{
Modelling the Structure of the Ringed Spiral NGC 4736
}

\author{
Wim van Driel \\ Nançay Radio Astronomy Observatory, Observatoire de Paris, France
}

Pieter Mulder

Kapteyn Astronomical Institute, Groningen University, The Netherlands

Françoise Combes

DEMIRM, Observatoire de Paris, France

\begin{abstract}
We studied the ringed RSab(r)-type spiral NGC 4736, which has a probably slightly oval disk and a very small bar. We mapped the galaxy in the $\mathrm{HI}$ and $\mathrm{H} \alpha$ spectral lines and we obtained long-slit optical spectra. These data were modeled using a 2-D gas dynamical code. The 2-D potential used is axisymmetric in the inner and outer regions and oval $(b / a=0.8)$ at intermediate radii only. The oval component rotates at a pattern speed of $40 \mathrm{~km} \mathrm{~s}^{-1} \mathrm{kpc}^{-1}$, close to the observed value. Inner and outer rings, like those observed, form at the inner and outer Lindblad resonances, though they co-exist only during a limited time interval in the simulations. The morphology and kinematics of the inner ring and spiral structure as observed in neutral and ionized hydrogen can be well understood in terms of gas dynamical simulations, given the form of the (stellar) potential. What remains to be explained is the origin of the nonaxisymmetric features in the mass distribution defining the potential.
\end{abstract}

\section{NGC 4736 - a Ringed Spiral with a Small Bar}

NGC 4736 is a nearby $\left(D=6.6 \mathrm{Mpc}, H_{0}=50 \mathrm{~km} \mathrm{~s}^{-1} \mathrm{Mpc}^{-1}\right)$ Sab-type spiral with an optical inner and outer ring. Its optical morphology indicates that the disk may be slightly oval, but there is no bar discernible in visible light.

We determined the morphology and kinematics of various components in its disk and compared them to gas dynamical model simulations, in order to study the origin of the spiral and ring patterns in this isolated, apparently non-barred system (see Mulder \& van Driel 1993, Mulder 1995a, 1995b; Mulder \& Combes 1995).

Morphologically, five annular regions can be distinguished in NGC 4736 :

- bright central region $\left(R<15^{\prime \prime}\right)$;

- inner spiral arms, bounded by an inner ring on the outside $\left(R \sim 50^{\prime \prime}\right)$;

- outer spiral structure $\left(R \sim 50^{\prime \prime}-210^{\prime \prime}\right)$; 
- very low surface brightness region, and

- faint outer ring $\left(R \sim 330^{\prime \prime}\right)$.

A Small Non-Axisymmetric Component: Since we started our studies, a small ( $\sim 12^{\prime \prime}$ long) bar has been found on near-infrared images, which indicates the existence of a non-axisymmetric component in its potential.

Our $R$-band CCD image also clearly shows an ellipsoid structure in the center, after subtraction of the rotationally symmetric component. It has a position angle roughly perpendicular to that of the outer isophotes.

\section{Observations - a Multiwavelength Study}

We obtained the following optical and radio observations of the galaxy:

- CCD broad-band: inner regions in $B, V$, and $R$ (La Palma $1.0 \mathrm{~m}$ );

- CCD broad-band: entire galaxy in $R$ and $I$ (Kiso $1.0 \mathrm{~m}$ Schmidt);

- H $\alpha$ line: imaging Fabry-Pérot, inner regions (La Palma $2.5 \mathrm{~m}$ );

- stellar absorption lines: long-slit spectra (La Palma $2.5 \mathrm{~m}$ );

- 21-cm HI line imaging: entire galaxy (Westerbork), and

- $\mathrm{CO}(1-0)$ and $\mathrm{CO}(2-1)$ line imaging: inner region (IRAM).

We thus obtained detailed maps of the distribution and kinematics of several galaxian constituents - neutral gas, ionized gas and stars. The galaxy has been mapped in the $\mathrm{CO}(1-0)$ and $\mathrm{CO}(2-1)$ lines by Gérin et al. (1991).

\section{Observations - Results}

Judging from surface photometry, NGC 4736 probably has an intrinsically slightly oval disk. Our studies cannot resolve the uncertainty about the inclination of the disk or other components in the galaxy. This causes uncertainty in the derivation of rotation curves under the assumption of circular rotation, as well as in dynamical model calculations (see below). A highly non-axisymmetric stellar morphologic component is seen in its inner ca. $10^{\prime \prime}$ of radius, but only in the red and near-infrared.

Kinematic Anomalies - Oval Distortion: Kinematic anomalies in NGC 4736, reported earlier in the literature (Buta 1988), are small: deviations from circular rotation are below half of the FWHM velocity resolution $\left(\sim 30 \mathrm{~km} \mathrm{~s}^{-1}\right)$ of our $\mathrm{HI}$ and $\mathrm{H} \alpha$ data. A good correspondence can be achieved between observed $\mathrm{H} \alpha$ and HI velocities and gas dynamical simulation results (see below) by fine tuning the mass-to-light ratio in the simulations and the kinematic major axis position angle used in the projection of simulation results on the sky plane.

In the inner regions of the galaxy, kinematic and morphologic major axis position angles of the stars are about $10^{\circ}$ lower than those of the gas components. 
For each component the kinematic major axis position angle is lower, again by some $10^{\circ}$, than the morphologic value. These differences indicate that the potential of this object is non-axisymmetric.

\section{Gas Dynamical Models - Fitting the Features}

We compared our observational data on the various gaseous components (ionized, neutral atomic and neutral molecular) to existing results of gas dynamical model calculations of the galaxy (Gérin et al. 1991), augmented with similar higher resolution models.

The Model-Basics: The 2-D potential used is axisymmetric at $R<16^{\prime \prime}$ and $R>180^{\prime \prime}$ (i.e., at $<0.5$ and $>5.5 \mathrm{kpc}$ ) and it is oval, with $b / a$ of 0.8 , at intermediate radii only. The oval component is introduced gradually during the first $200 \mathrm{Myr}$ of the simulations, which span a total of $4.5 \mathrm{Gyr}$. It rotates at a pattern speed of $40 \mathrm{~km} \mathrm{~s}^{-1} \mathrm{kpc}^{-1}$, close to the observed value.

Inner and outer rings resembling those actually observed are seen to form at the inner and outer Lindblad resonances, but they co-exist only during a limited time interval, i.e., between 2-3 Gyr (2-20 rotations of the oval disk) after the start of the simulation. The best global agreement in morphology with the observations is reached after some 18 rotations, when the model shows both rings at the right radius, and no structure not present in the data.

The Model-Fine Tuning: Having chosen this "morphologically correct" model, it was fine tuned using kinematic data ( $\mathrm{HI}$ and $\mathrm{H} \alpha$ velocity fields,) of the inner ring. Good correspondence was found for an inclination of $35^{\circ}$ and a kinematic major axis p.a. of $115^{\circ}$. The (observed-minus-calculated) velocity residual maps show significant differences of order $40 \mathrm{~km} \mathrm{~s}^{-1}$ (i.e., $\lesssim 2$ times the velocity resolution) on the SW side of the inner ring only, and these differences are $\lesssim$ the velocity resolution of the data.

\section{References}

Buta, R. J. 1988, ApJS, 66, 233

Gérin, M., Casoli, F., \& Combes, F. 1991, A\&A, 251, 32

Mulder, P. S. 1995a, Ph. D. thesis, Groningen University, The Netherlands

Mulder, P. S. 1995 b, A\&A, in press

Mulder, P. S. \& van Driel, W. 1993, A\&A, 272, 63

Mulder, P. S. \& Combes, F. 1995, A\&A, in press 